The ion temperature evolution on TCA during Alfven wave heating and in non-stationary Ohmic conditions

This article has been downloaded from IOPscience. Please scroll down to see the full text article.

1989 Plasma Phys. Control. Fusion 31527

(http://iopscience.iop.org/0741-3335/31/4/003)

View the table of contents for this issue, or go to the journal homepage for more

Download details:

IP Address: 128.178.125.142

The article was downloaded on 18/02/2013 at 08:39

Please note that terms and conditions apply. 


\title{
THE ION TEMPERATURE EVOLUTION ON TCA DURING ALFVÉN WAVE HEATING AND IN NON-STATIONARY OHMIC CONDITIONS*
}

\author{
A. De Chambrier, B. P. Duval, J. B. Lister, F. J. Mompeán † \\ and J.-M. MORET \\ Centre de Recherches en Physique des Plasmas, Association Euratom-Confédération Suisse, \\ Ecole Polytechnique Fédérale de Lausanne, 21, Av. des Bains, CH-1007 Lausanne, Switzerland
}

(Received 18 July 1988; and in revised form 30 September 1988)

\begin{abstract}
High power Alfvén Wave Heating has been applied to the TCA Tokamak and results in an electron and ion temperature increase, together with a plasma density rise. The various terms leading to an ion temperature increase are investigated to determine whether any of the applied r.f. energy is absorbed directly by the ions. This is done by a detailed comparison with non-stationary Ohmic discharges. We conclude that although a large fraction of the ion temperature increase may be assigned to the density increase, there remains a component that is most simply described by a direct ion heating.
\end{abstract}

\section{INTRODUCTION}

THE APPLICATION of Alfvén Wave Heating to the TCA Tokamak plasma has invariably led to an increase in the ion temperature. Alfvén Wave theory predicts that the r.f. power will be absorbed by the electrons and thus we would expect any ion heating to result from electron-ion collisions. Analysis of the ion temperature increase is confused by the significant increase in the plasma density induced by the r.f. power (APPERT et al., 1986). This paper presents an analysis of the ion temperature increase, using empirically calibrated models to correct for the effect of the density change and any observed change in the electron temperature to determine if our theoretical perceptions are correct.

The results described were obtained on the TCA Tokamak (CHEETHAM et al., 1981) $\left(R, a=0.61 \mathrm{~m}, 0.18 \mathrm{~m}, I_{\mathrm{p}}\right.$ up to $170 \mathrm{kA}, B_{\phi}$ up to $1.51 \mathrm{~T}$, filling gas hydrogen or deuterium). In the reported experiments, additional r.f. heating was provided at a frequency of 2.0-2.5 Mhz with pulses up to $80 \mathrm{~ms}$ long with a delivered r.f. power of up to $200 \mathrm{~kW}$. The details of the Alfvén Wave launching scheme are described in detail by ColLins et al. (1986) and a resume of its application to TCA has been given by Besson et al. (1986), and so here, we shall simply recall the relevant features of this heating scheme. A spatial resonance structure is driven within the plasma at a minor radius, $r$, with a toroidal magnetic field $B_{\phi}$ and with an ion cyclotron frequency of $\omega_{\mathrm{ci}}$, at which the condition:

$$
\omega^{2}(r)=\left(1 / R^{2}\right)(n+m / q(r))^{2} \cdot\left(B_{\phi}^{2} / \mu_{0} \rho(r)\right) \cdot\left(1-\omega^{2} / \omega_{\mathrm{ci}}^{2}\right)
$$

is satisfied. $\rho(r)$ and $q(r)$ are the local mass density and safety factor, and $\omega$ is defined

* This paper is an expanded version of material which originally was a contributed presentation at the 15th EPS Plasma Physics Division Conference, Cavtat, Yugoslavia, May 1988.

$\uparrow$ Seconded from C.I.E.M.A.T., Madrid, Spain. 
by the excitation frequency, $f_{\text {gen }}=\omega / 2 \pi ;(n, m)$ are the toroidal and poloidal mode numbers of the excited structure [in our notation, $(N, M)$ represents the driving antennae structure and $(n, m)$ the mode driven in the plasma].

At the resonant layers, the wave from the antennae is converted and then propagates towards the plasma core as the Kinetic Alfvén Wave. This has been experimentally confirmed by a direct measurement of the associated density fluctuations (BEHN $e t$ al., 1987). The energy in the Kinetic Alfvén Wave is theoretically predicted to be dissipated by Electron Landau Damping since its wave speed corresponds to an electron with a kinetic energy of $100 \mathrm{eV}$, typical of our plasma outer region. There is no theoretical prediction of a significant direct transfer of energy to the ions, which are, of course, much slower. Our measurement of the launched r.f. power is deduced by subtracting the power launched during a shot without gas from that with a plasma. This so-called plasma antenna loading is normally a significant fraction of the total loading, but we may only reasonably assume that this measured power enters the plasma. In the same way we reasonably assume that this power is absorbed near the resonance layers by the electrons, and investigate if our energy balance is consistent with this.

To measure the plasma ion temperature from the spectrum of emitted neutrals, we used a five channel neutral particle analyser (N.P.A.) obtained from the A.F. IOFFE Institute (AFROSIMOV et al., 1975). The installation, calibration and operation of this spectrometer on TCA are described in detail by De CHAMBRIER (1987). Most of the data were obtained with the N.P.A. flight line perpendicular to, and in the same plane as the major axis. The spectrometer was equipped with a pivotable flight line to the Tokamak which was used to scan the spectrometer acceptance cone across the plasma minor radius. The spatial profile of the observed ion velocity distribution was thus investigated.

The data in Fig. 1 illustrate the physical problem discussed in this paper. The r.f. pulse produces a rapid increase in $\left(\beta+l_{\mathrm{i}} / 2\right)$, the soft X-ray flux, the plasma density, and the flux in the N.P.A. channels. For certain plasma conditions, the ion temperature measured by the N.P.A. increases significantly (De CHAMBRIER, 1987). The understanding of this temperature rise is complicated by the increasing plasma density, so the plasma may not be considered to be quasi-stationary. Specifically, as the plasma density increases, the increased collisionality raises the electron-ion power transfer, which, even for Ohmic discharges, leads to an increase in the ion temperature. An early effort to estimate this indirect heating effect, by plotting the plasma temporal evolution in the $n_{\mathrm{e}}: T_{\mathrm{i}}$ plane (BeHN et al., 1984), suggested that the increase in the ion temperature was greater than expected simply from the density increase. Before we can determine the size of the effect, which we may loosely describe as a direct r.f. heating term, the contribution of the increasing density to the ion temperature must be accurately modelled and its effect taken into account.

The timescale of the density increase (Fig. 1) is such that a quasi-stationary analysis is inadequate, obliging us to include, in the power balance, terms depending on the time derivatives of both density and ion temperature. Section 2 of this paper describes non-stationary Ohmically heated discharges, providing us with a model with which we can compare the r.f. heated discharges. Section 3 gives a general survey of the r.f. heated results, comparing the evolution of those discharges with equivalent Ohmically heated discharges. In Section 4 the r.f. excitation mode is varied and we look for 

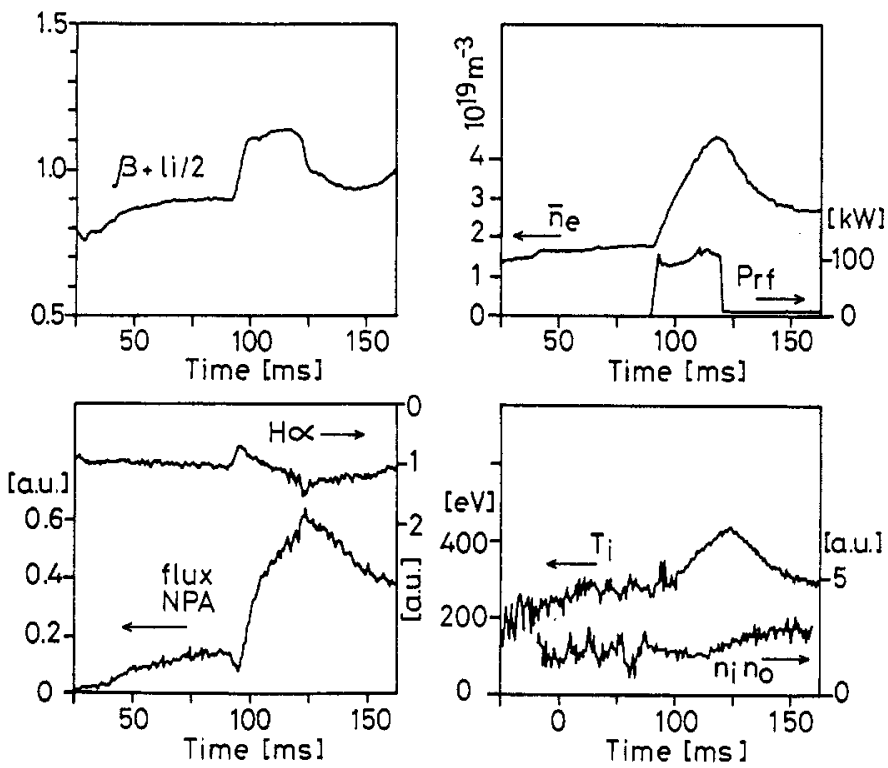

FIG. 1.-Typical evolution on application of the r.f. pulse. The plasma energy content, plasma density and $\mathrm{H} x$ traces are shown together with the temporal evolution of the ion temperature, the N.P.A. flux and the deduced $n_{n} n_{0}$ product.

effects due to the positions of the resonance surfaces. In Section 5 we try to circumvent the non-stationary analysis which, in Section 3 , involved a wide range of densities, by applying the technique of r.f. power modulation. All of these three sections will conclude that there exists a non-understood, but non-zero, missing element in the ion power balance, summarized in Section 6.

\section{STUDY OF OHMICALLY HEATED DISCHARGES}

In the Introduction, we showed that it is necessary to predict the temporal evolution of the ion temperature for a rapidly evolving electron density. Only then will we be able to determine if the ions are heated only by the increased collisionality or whether there is another mechanism involved. The N.P.A. was carefully set up with special attention paid to the ion temperature calculation and in an estimation of the experimental errors. This is discussed in detail by DE CHAMBRIER (1987). To summarize this procedure, Fig. 2 shows the neutral particle energy spectrum obtained from a series of reproducible Ohmic discharges. The lines join the data from the spectrometer channels for a single discharge, and each line corresponds to a different deflection plate voltage, which changes the spectrometer energy dispersion. The ion temperature is determined by the slope of the points, and the alignment of the data in Fig. 2 allows us to be confident that the experimental procedure is correct.

We analysed plasmas in varied experimental conditions. Analysis of quasi-stationary Ohmic data $\left(\mathrm{d} n_{\mathrm{e}} / \mathrm{d} t\right.$ and $\mathrm{d} T_{\mathrm{i}} / \mathrm{d} t$ small) shows a strong systematic dependence of the ion temperature on both plasma density and plasma current with deuterium as the filling gas (Fig. 3). The ion temperatures are lower than those predicted by the Artsimovitch scaling (solid curves) and saturates at high current and density. This strong current dependence led us to reject data in which the plasma current was not 


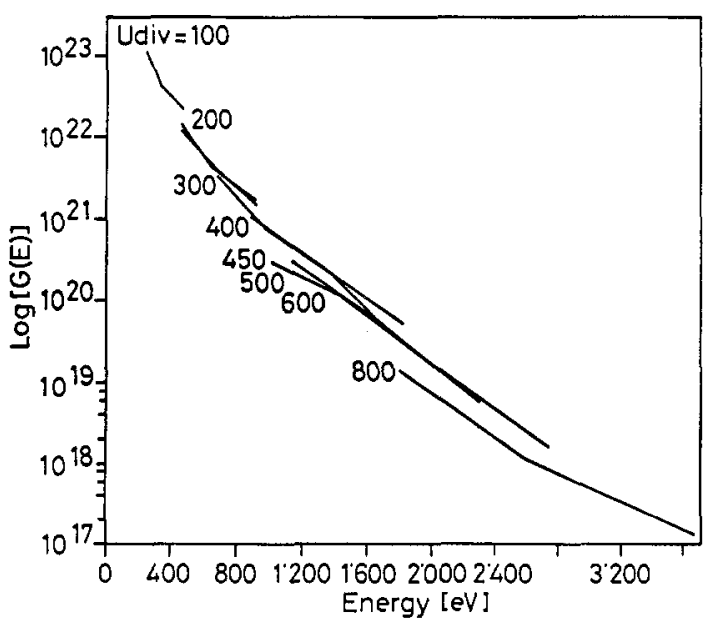

FIG. 2.-The normalized energy spectrum $G(E)$ versus particle energy for all five N.P.A. channels for a sequence of reproducible discharges in which the divider voltage, $U_{\mathrm{div}}$, was changed. The channel selection energies are proportional to $U_{\mathrm{div}}$ and the ion temperature is deduced from the slope of this graph. The reproducibility of the slope with varying $U_{\text {div }}$ demonstrates the correct calibration of the N.P.A.

held constant during the experiment. The ion temperature depends on the ion mass, and also on the ratio between electron and ion temperatures shown in Fig. 4. At high density, $T_{\mathrm{e}} / T_{\mathrm{i}}$ approaches unity in hydrogen, at which point the estimate of the collisional power transfer $\left(P_{\mathrm{ei}}\right)$, will be unusably inaccurate. We start with the general expression for $P_{\mathrm{ei}}$ :

$$
P_{\mathrm{ei}}=12.2 \times 10^{5} n_{\mathrm{e} 19}^{2}\left(T_{\mathrm{e}}-T_{\mathrm{i}}\right) T_{\mathrm{e}}^{-3 / 2} A^{-1} \quad\left(\mathrm{~W} \mathrm{~m}^{-3}\right)\left(\mathrm{m}^{-3}, \mathrm{eV}\right)
$$

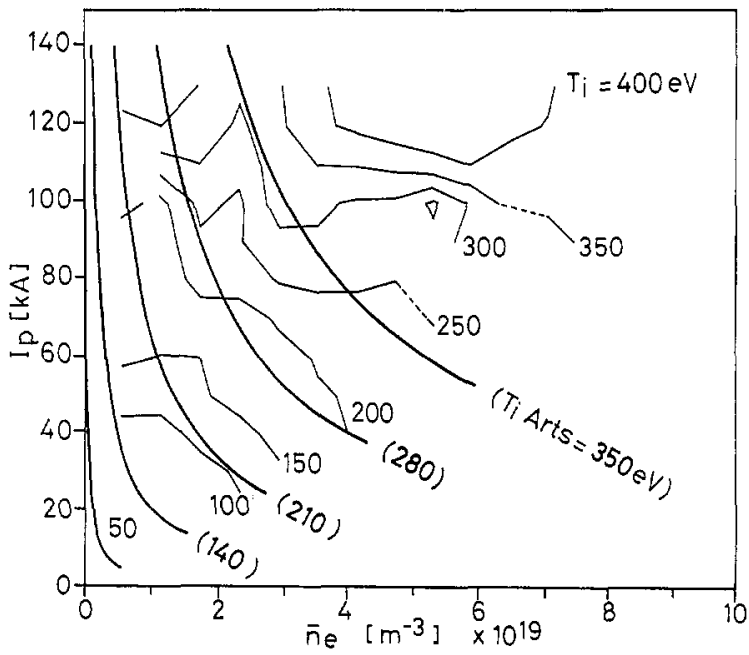

FIG. 3.-The dependence of ion temperature on plasma density and plasma current $\left(B_{\phi}=1.5\right.$ $\mathrm{T}$, deuterium plasma). The measured ion temperatures are lower than that predicted by the Artsimovitch scaling law shown by the bold lines. 


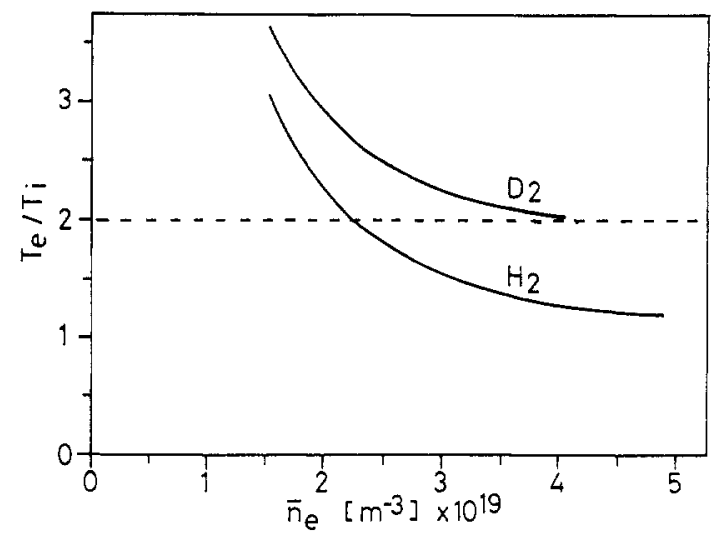

FIG. 4.-The ratio $T_{\mathrm{e}} / T_{\mathrm{i}}$ for hydrogen and deuterium as a function of electron density, where the ions are considered to be heated solely by collisions with electrons.

where $n_{\mathrm{e} 19}$ is the plasma density in units of $10^{19} \mathrm{~m}^{-3}$. In the experimental conditions in which most of the analyses were performed on deuterium discharges, $P_{\mathrm{ei}}$ is relatively insensitive to the value of the electron temperature, and we may use equation (2) together with $T_{\mathrm{i}}=T_{\mathrm{e}} / 2$ obtaining:

$$
P_{\mathrm{ei}} \sim 4.5 \times 10^{5} \cdot n_{\mathrm{e} 19}^{2} \cdot T_{\mathrm{i}}^{-1 / 2} \cdot A^{-1}\left(\mathrm{~W} \mathrm{~m}^{-3}\right)\left(\mathrm{m}^{-3}, \mathrm{eV}\right) .
$$

If we restrict ourselves to quasi-stationary conditions, and to one plasma current, $q_{a} \sim 3.1$, we find that a low order polynomial (quadratic) enables us to obtain a close approximation to the density dependence of the ion temperature for a wide range of different plasma conditions (Fig. 5). For quasi-stationary conditions, this adequately predicts the discharge locus in the $n_{\mathrm{e}}: T_{\mathrm{i}}$ plane.

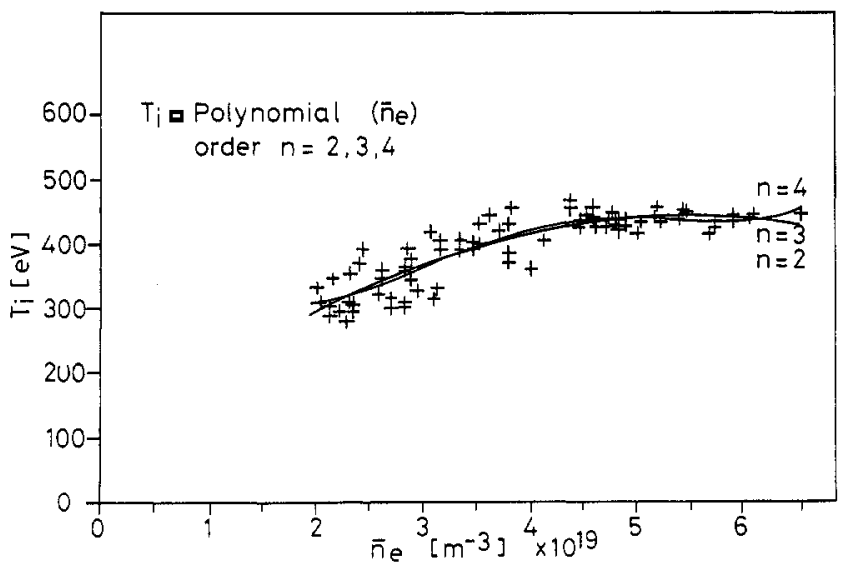

FIG. 5.- The density dependence of the ion temperature expressed as polynomials of order 2,3 , and 4 . The quadratic approximation is clearly sufficient and was subsequently used to model the r.f. discharges. Note: this figure shows data with many different experimental conditions, and for a reproducible series of discharges the scatter is typically reduced by at least a factor of 4 . 


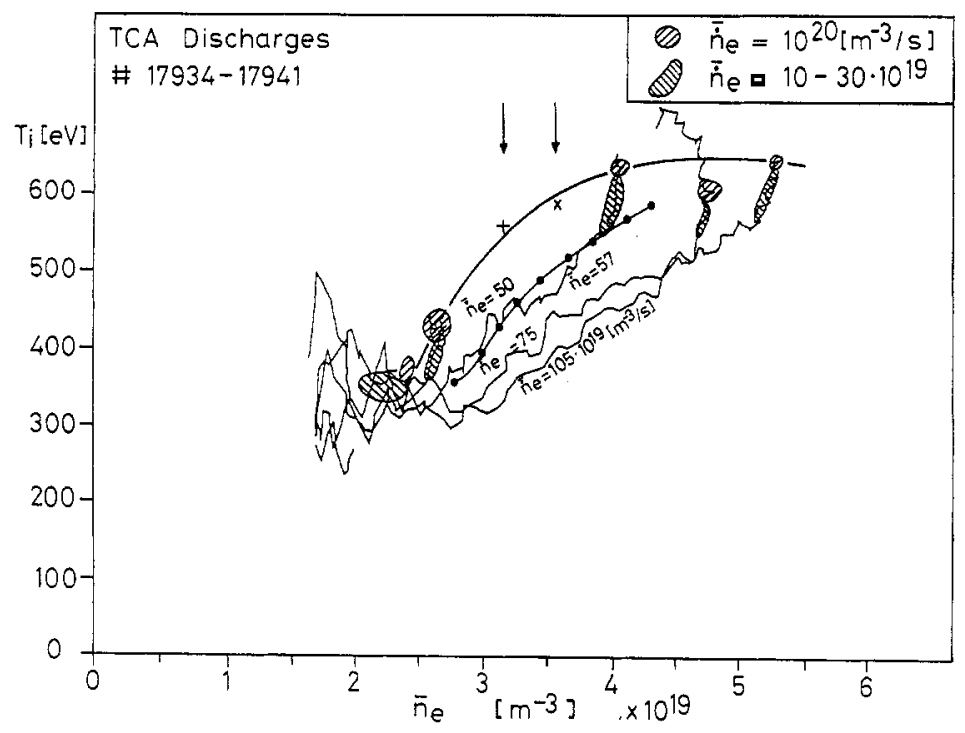

FIG. 6,-Comparison between the stationary dependence (smooth curve) and non-stationary evolutions in the $T_{\mathrm{i}}: n_{\mathrm{e}}$ plane $\left(B_{\phi}=1.5 \mathrm{~T}, q_{a}=3.1\right)$. The vertical arrows indicate the plasma density corresponding to a DAW resonance, and the shading indicates regions where the value of $\mathrm{d} n_{\mathrm{e}} / \mathrm{d} t$ changed during the non-stationary evolutions.

Conversely, non-stationary discharges track different trajectories in the $n_{\mathrm{e}}: T_{\mathrm{i}}$ plane, depending on the time derivative of the electron density (Fig. 6). An increase in the temporal density derivative, $\mathrm{d} n_{\mathrm{e}} / \mathrm{d} t$, gives an lower ion temperature than observed for quasi-stationary conditions (Fig. 7). Since large negative values of $\mathrm{d} n_{\mathrm{e}} / \mathrm{d} t$ could not be achieved by cutting the gas feed, only fast increases for a positive $\mathrm{d} n_{\mathrm{e}} / \mathrm{d} t$ were investigated. The linear dependence of $T_{\mathrm{i}}$ allows us to extrapolate to $\mathrm{d} n_{\mathrm{e}} \mathrm{d} t=0$. The ion temperature thus obtained (around $500 \mathrm{eV}$ ) is less than 20\% different from the parametric description of stationary discharges in Fig. 5.

The dependence of $T_{\mathrm{i}}$ on $\mathrm{d} n_{\mathrm{e}} / \mathrm{d} t$ is weaker than predicted from the supposition that

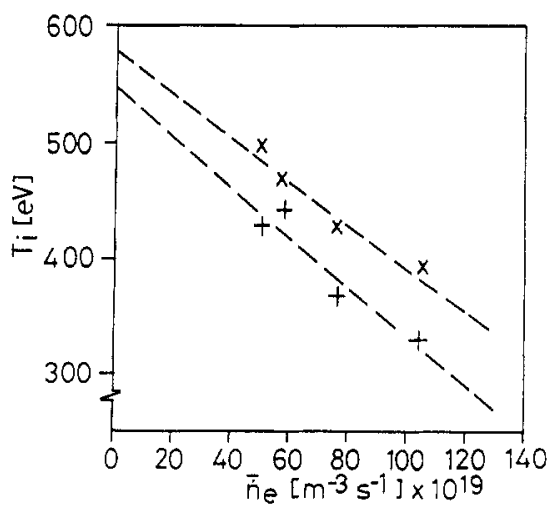

FIG. 7.-Dependence of the ion temperature on the rate of increase of electron density for two densities indicated by $\times$ and $+\left(B_{\phi}=1.51, q_{a}=3.1\right)$. 
$\Delta W_{\mathrm{i}}=0$. Firstly, both the ion confinement and the collisional energy transfer increase with increasing density, as discussed below. Secondly, the increased density at the centre also corresponds to a convected ion energy input, equal to $\mathrm{d} n_{\mathrm{c}} / \mathrm{d} t \cdot T_{\mathrm{i}}^{*}$, where $T_{1}^{*}$ is an equivalent cold ion influx temperature. We cannot go further with this analysis without a full set of radial profile data, but the present aim is only to provide an empirical model to describe the measured $T_{\mathrm{i}}$ value.

We see from Fig. 6 that the measured value of $T_{\mathrm{i}}$ can exceed the quasi-stationary state value when the gas valve that provokes the density increase is shut. Since $P_{\mathrm{ei}}$ will be calculated to be smaller in this case, the apparent ion energy confinement time will be greater at this instant. Due to the end of the current plateau in TCA, it is not clear whether this higher confinement time can be maintained for long enough to be significant.

A rather simplified ion power balance can be written, valid for an ill-defined central region, as:

$$
\begin{aligned}
\mathrm{d} W_{\mathrm{i}} / \mathrm{d} t & =3 / 2 e\left(\mathrm{~d} T_{\mathrm{i}} / \mathrm{d} t n_{\mathrm{e}}+T_{\mathrm{i}} \mathrm{d} n_{\mathrm{e}} / \mathrm{d} t\right) \\
& =P_{\mathrm{ei}}-3 / 2 e T_{\mathrm{i}} n_{\mathrm{e}} / \tau_{E \mathrm{i}}
\end{aligned}
$$

where $W_{\mathrm{i}}$ is the energy density of the ions in this region, and, for the moment, all losses have been lumped together using an ion energy confinement time $\tau_{E:}$. The observed linear dependence is included in the assumption that $P_{\mathrm{ei}}$ and $\tau_{E \mathrm{i}}$ do not depend on $\mathrm{d} n_{\mathrm{e}} / \mathrm{d} t$, giving a lag on $\mathrm{d} T_{\mathrm{i}} / \mathrm{d} t$ equal to $T_{\mathrm{i}} \cdot \mathrm{d} n_{\mathrm{e}} / \mathrm{d} t / n_{\mathrm{e}}$. The depressed value of $T_{\mathrm{i}}$ for high $\mathrm{d} n_{\mathrm{e}} / \mathrm{d} t$ corresponds to the time integral of this lag.

These results now give us the confidence to solve the ion-temperature evolution given by equation (4). In our model, we chose an empirical parametrization: $\tau_{E \mathrm{E}}\left(n_{\mathrm{e}}\right)=C n_{\mathrm{e}}^{a}$ in which $C$ and $a$ were calculated to reproduce the quasi-stationary form of $T_{\mathrm{i}}\left(n_{\mathrm{c}}\right)$. The results of this model, together with the experimental data, are plotted in Fig. 8, showing the excellent agreement. In this analysis, the parameters were deduced from an Ohmic discharge from the same discharge series for which TCA is very reproducible.

The possibility of additional impurities during the r.f. pulse was also considered in detail by DE CHAMBRIER (1987). The effect of impurity ions on the electron-hydrogen energy transfer was calculated and compared with experiments using puffs of different inpurity gases into an established Tokamak plasma. The results indicated that an impossibly high impurity density would be required to alter the pure gas analysis significantly, the upper limit on the Tokamak impurity density having been previously determined from a multi-bolometer diagnostic (JOYE et al., 1986).

In conclusion, our parametrization of the Ohmic data leads to a zero-dimensional evolution which accurately reproduces gas-puff produced density rises with a similar temporal density evolution as those encountered during the r.f. heating experiments. On closing the gas feed, the ion temperature can exceed the quasi-stationary value for the given density.

\section{SURVEY OF THE ION TEMPERATURE INCREASE DURING r.f.}

Before we discuss the analysis of the r.f. heated plasmas, it is useful to describe the phenomology of the N.P.A. measurements during Alfvén Wave Heating. Applying 


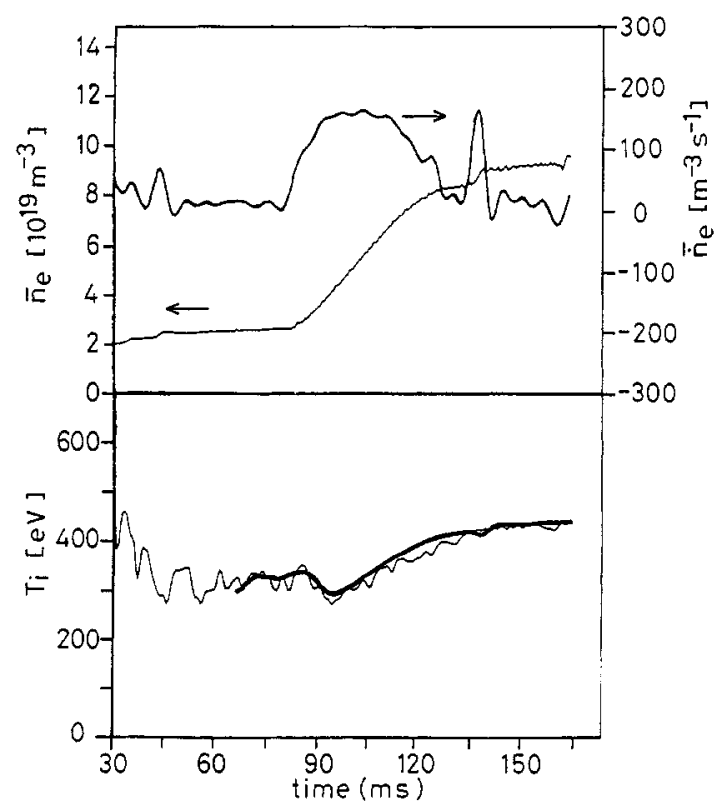

FIG. 8.-Evolution of the ion temperature during gas-puffing in an Ohmically heated discharge, comparing the raw data and the evolution model using the empirical coefficient from Fig. $5\left(B_{\phi}=1.51, q_{a}=3.1\right)$.

the r.f. pulse, as seen in the Introduction, leads to an increase of the signals in all the N.P.A. channels and in the estimated ion temperature (Fig. 1). Figure 9 shows the measured neutral particle energy spectrum before, during and after an r.f. heating pulse. The neutral particle energy spectrum is Maxwellian in all the cases showing that we may consider that the spectrometer measures an ion temperature through the r.f. pulse.

A radial profile of the ion temperature distribution was performed using the pivot-

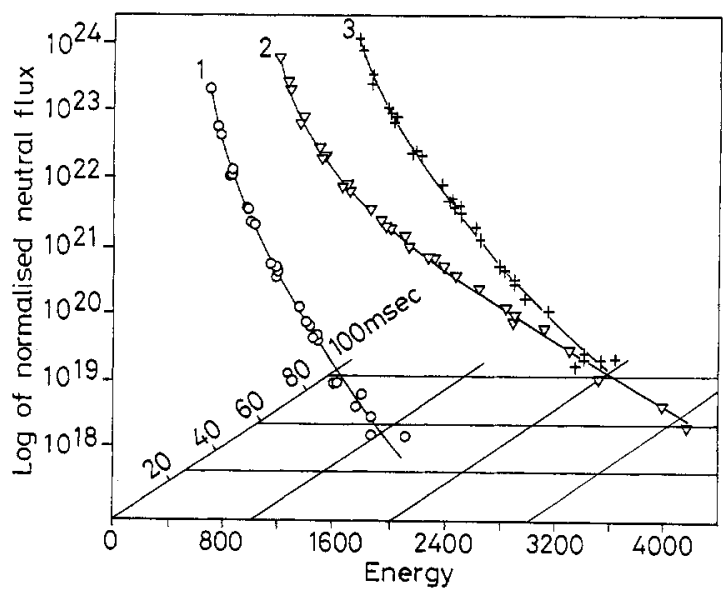

FIG. 9.-The neutral particle energy spectrum (1) before the r.f. pulse, (2) during it and (3) following it. The spectrum is found to be Gaussian in all three cases. 
able flight line. These profile measurements were performed for three plasma densities corresponding to different spectral conditions (Fig. 10). All three profiles are quite flat, which allows us to conclude a large plasma centre with a fairly constant ion temperature. We use this observation in the numerical analysis below, to justify a global ion energy balance to such a homogeneous central plasma region. The majority of our experimental observations were thus performed with the N.P.A. on the central chord which was assumed to be a measurement of this central region. All these observations make us confident in concluding that the observed ion temperature increase is real.

The ion temperature increase is observed to be not only a function of the r.f. power, but also the toroidal field, $B_{\phi}$, plasma current, $I_{\mathrm{p}}$ and the plasma density, $n_{\mathrm{e}}$. It is largest for low toroidal field, low density and low plasma current. The effect of $B_{\phi}$ has not been fully investigated, but may be explained by the related reduction in both $I_{\mathrm{p}}$ and $n_{\mathrm{e}}$, either of which would lead to a larger ion temperature increase, simply from the relative increase in the equilibration power.

As a function of the r.f. power, the ion temperature rises linearly at low power levels, but saturates at higher power where the density rise is considerable. To parameterize this saturation, the maximum ion temperature obtained for three different plasma currents was evaluated for different plasma densities at fixed $B_{\phi}(1.5 \mathrm{~T})$ and a narrow range of r.f. powers (85-130 kW) (Figs 11a,b,c). Due to the accompanying $n_{\mathrm{e}}$ rise, the maximum ion temperatures before and during the r.f. pulse for a given $n_{\mathrm{e}}$ are obtained from different discharges. The maximum ion temperature is $300-350 \mathrm{eV}$, with the increase being greatest for discharges with the lowest pre-r.f. ion temperature. However, at this high density, the ion temperature with r.f. is not higher than the achieved Ohmic value except for $q_{a}=2.3$, where the Ohmic temperature is depressed.

To explain this ion temperature saturation qualitatively, with plasma conditions $n_{\mathrm{c}}=5 \times 10^{19} \mathrm{~m}^{-3}$ and $n_{\mathrm{c}}(r=0) \sim 8 \times 10^{19} \mathrm{~m}^{-3}$, we start with equation (2) for the equilibration power, $P_{\mathrm{ei}}$. This would give an equilibrium power loss of $-0.2 \mathrm{~W} \mathrm{~cm}^{-3}$, for a value of $T_{\mathrm{i}} 100 \mathrm{eV}$ higher than $T_{\mathrm{e}}$, over and above the existing ion diffusion

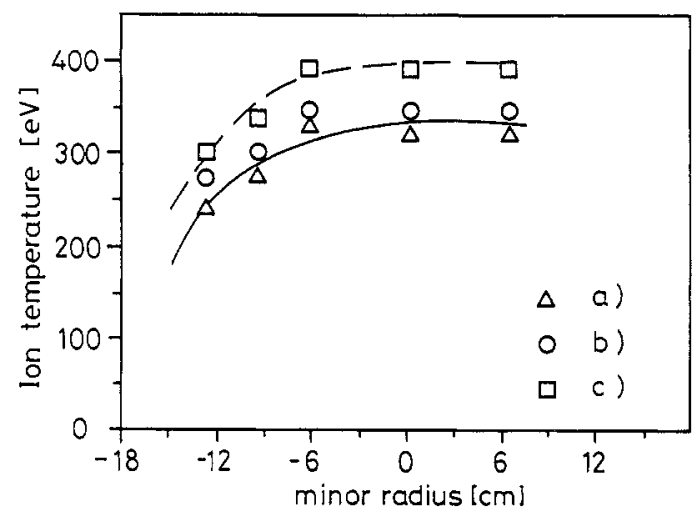

FIG. 10.-Plot of the N.P.A. ion temperature from a scan of the line-of-sight across the minor Tokamak radius at plasma densities corresponding to (a) just below the $(2,0) \mathrm{DAW}$, (b) on the $(2,0)$ DAW, and (c) just after the $(2,0)$ DAW resonance. In all cases, the ion profiles were found to be rather flat, allowing us to perform most of our measurements perpendicular to the machine. 


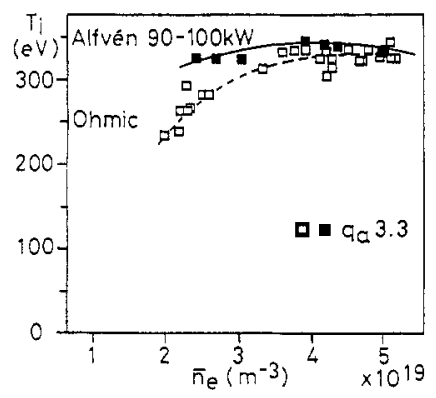

(a)

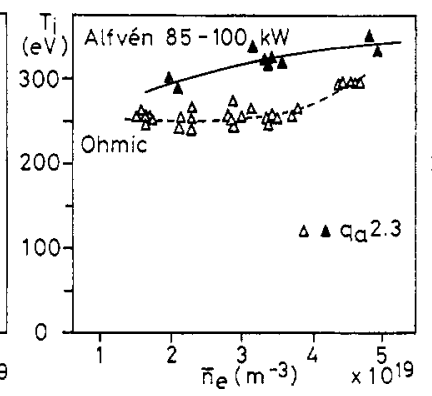

(b)

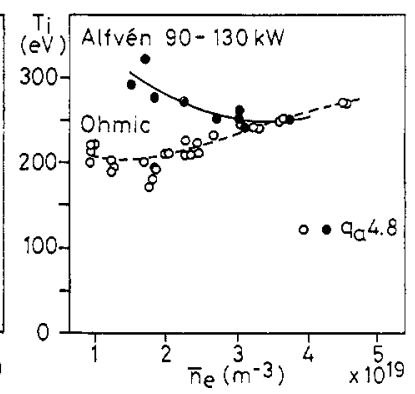

(c)

Fig. 11.-Ion temperature of the Ohmic and r.f, heated plasma for (a) $I_{\mathrm{p}}=122 \mathrm{kA}$, (b) $I_{\mathrm{p}}=166 \mathrm{kA}$, (c) $I_{\mathrm{p}}=80 \mathrm{kA}\left(B_{\phi}=1.5 \mathrm{~T}, P_{\text {r.f. }}\right.$ as shown). The solid lines represent the trends for other plasma parameter values. The error bar is seen from the scatter of the points at the same plasma and magnetic configuration conditions.

losses. The total ion power loss would then exceed the r.f. input power. This demonstrates why, at these densities, the ion temperature must saturate for a given r.f. power level. Put another way, only a direct ion heating efficiency of greater than $10 \mathrm{eV} \cdot 10^{19}$ $\mathrm{m}^{-3} \mathrm{~kW}^{-1}$ could counteract the reduction in collisional power transfer as the ion temperature increases towards $T_{\mathrm{e}}$. This argument could also explain why the ion temperature increase is largest when the initial temperature is much lower than the electron temperature.

The simplest analysis of the ion temperature rise with r.f., is a comparison with the Ohmic model described above. The simulated ion temperature evolution, using the measured $n_{\mathrm{e}}$ evolution, does not agree with the measured value of $T_{i}$, Fig. 12. The ion

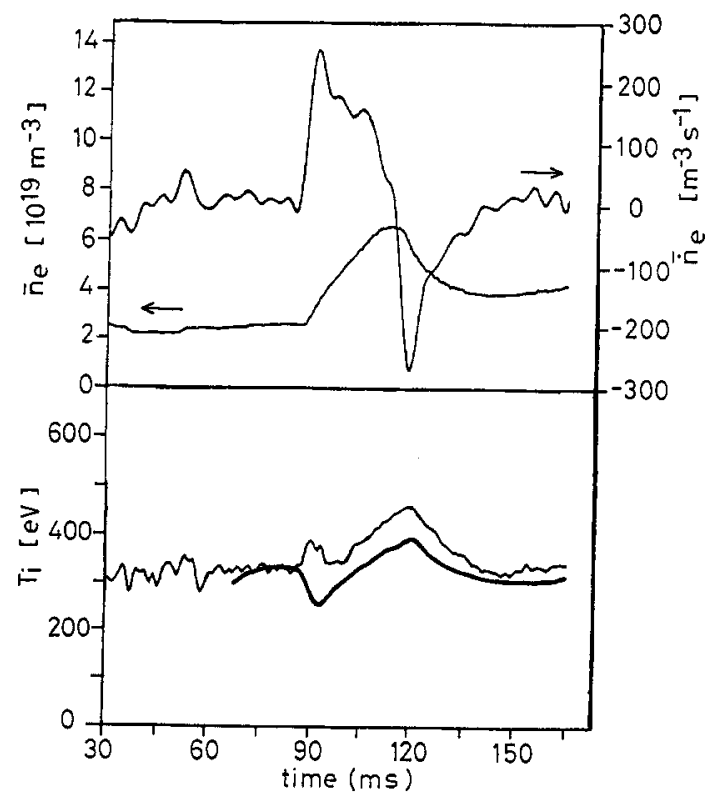

FIG. 12.-Comparison between measured and predicted ion temperature evolution during the r.f. pulse $\left(B_{\phi}=1.5 \mathrm{~T}, I_{\mathrm{p}}=122 \mathrm{kA}\right)$. In comparison with Fig. 8, with r.f., the model is in sharp disagreement with the measured ion temperature evolution. 
temperature increases at the start of the r.f. pulse, instead of the decrease which would be predicted by $\mathrm{d} W_{\mathrm{i}} / \mathrm{d} t \sim 0$, suggesting that the model is incomplete. Following this initial rise the ion temperature remains above the simulated value and diminishes after the end of the r.f. pulse to a non-zero increment.

In order to "correct" the simulation we would need to change or add at least one term in the ion power balance. We could:

(1) add a term labelled "direct ion heating" for which there is no theoretical support;

(2) modify $P_{\mathrm{ei}}$ by an anomaly factor which switched on during the r.f. pulse;

(3) suddenly reduce the ion losses, increasing $\tau_{E \text { i }}$ during the r.f. pulse;

(4) assume that the incoming plasma is convected at high temperature (e.g. an incoming ion particle energy of $150 \mathrm{eV}$ would remove the ion temperature drop observed with the onset of r.f. heating in Fig. 12).

Since in our analysis the ion temperature would be the result of all of these terms, we cannot distinguish between these possibilities. In conclusion, we see a term that has the effect of directly heating the ions which is not accounted for by our Ohmic model.

\section{EFFECT OF THE EXCITED r.f. SPECTRUM}

In the previous section we treated the data for one specific r.f. excitation condition, with toroidal mode number $n=2$. From equation (1), we see that as the density rises, the excited spectrum changes, described by BESsON et al. (1986). Since we see an r.f. induced change in the ion power balance, we might expect some spectral r.f. dependence on this change.

This was studied by driving three r.f. excitation structures with $M=1$ (Collins et al., 1986), and by varying the working gas. Figure 13 shows the ion temperature evolution through a discharge in which the electron density evolves through the r.f. spectral condition corresponding to the threshold of a Discrete Alfvén Wave, DAW, signalled by a sharp peak in the antenna loading curves. The DAW is a global Alfvén r.f. resonance whose effect is observed on many plasma diagnostics and occurs at a slightly lower density than the continuum resonance, described by equation (1), with the same wave numbers (Collins et al., 1986). The increase in the ion temperature with electron density often changes at a DAW, e.g. in Fig. 13 it decreases after the DAW, although the electron density continues to rise. In other examples, the form of the density increase can also change at this point, which in extreme cases can lead to a plasma disruption. As opposed to the Ohmic discharges, the maximum ion temperature is no longer always obtained at the maximum value of plasma density.

To determine the r.f. power dependence (and with the induced density rise, the r.f. spectral dependence), a series of discharges were performed with the same pre-r.f. plasma density (and hence initial spectral condition), and a range of r.f. powers. To gather the results together, the maximum ion temperature and the maximum electron density observed during the r.f. pulse, not necessarily obtained simultaneously, are plotted. The r.f. power at which the density rose sufficiently to cross the next Alfven Wave continuum threshold is marked by a vertical arrow. We see that with the maximum density increasing approximately linearly, the ion temperature always tended to increase (Figs 14a,b,c). 


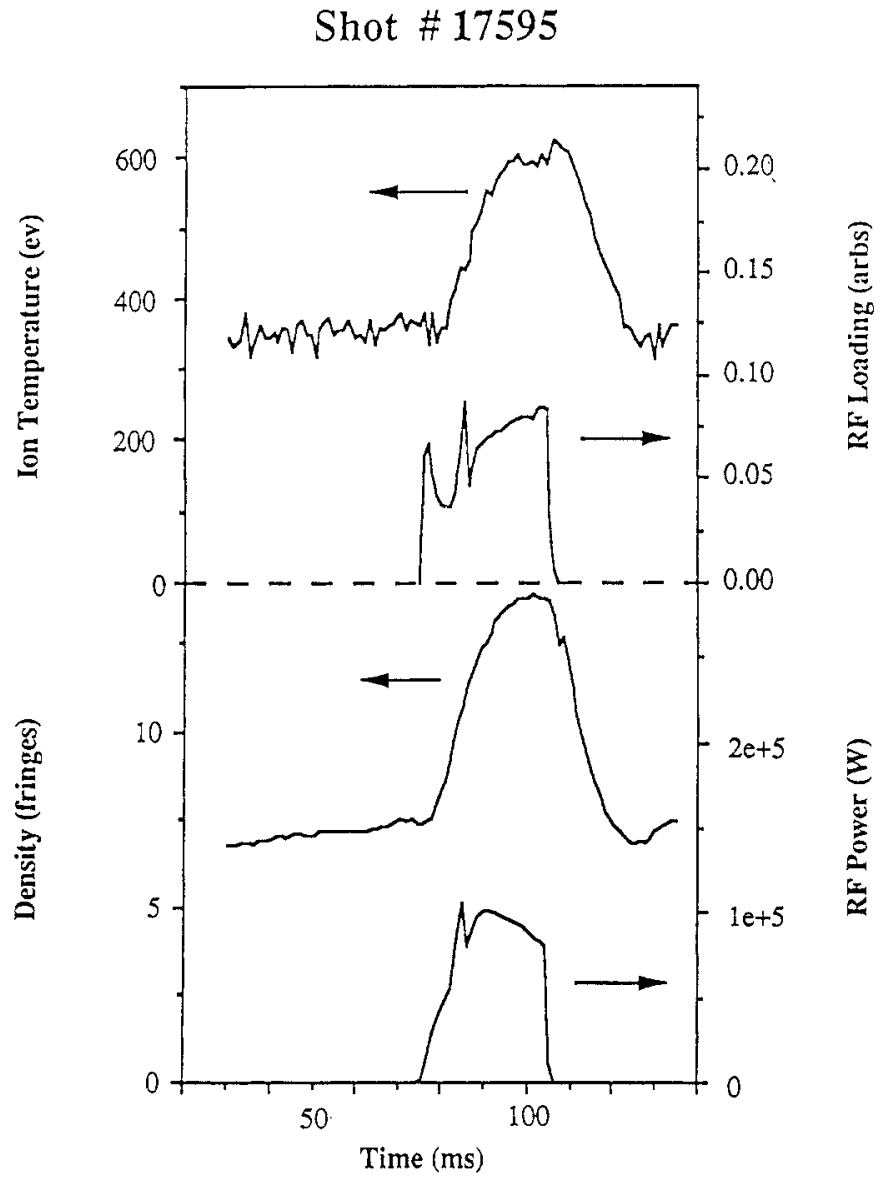

FIG. 13.-Example of a discharge which traverses a strong r.f. feature (the DAW) in which the density continues to rise whereas the ion temperature gradient exhibits a discontinuity.

Figure 14(a) $(N=1$, deuterium) shows a clear spectral effect. For the discharges above the critical power, the maximum ion temperature achieved actually decreased. The electron density, which increases approximately linearly with r.f. power, brings the discharge to the next threshold, after which the maximum ion temperature dropped with increasing r.f. power. This shows that the increase in the ion temperature can be a strong function of the position in the Alfvén Wave spectrum, and appears most "efficient" before a new continuum threshold which corresponds to the previous r.f. resonance layers being in the plasma edge. Once the new continuum threshold is crossed, the presence of the new central resonance surface results in a lower increase in $T_{\mathrm{i}}$. This behaviour is repeated in Fig. 14(c) $(N=4$, deuterium), once the $(N, M)=(4,-1)$ threshold is crossed. In both these cases, the ion temperature is well below the central electron temperature, measured by Thomson scattering and also indicated in the figures.

In contrast, Fig. 14(b) ( $N=2$, hydrogen) does not show this behaviour, and the maximum ion temperature carries on rising with increased r.f. power. This indicates 


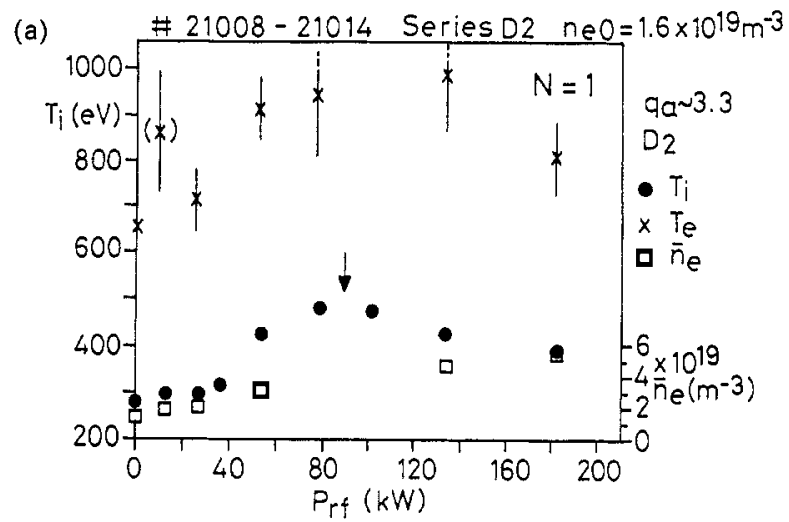

(b)

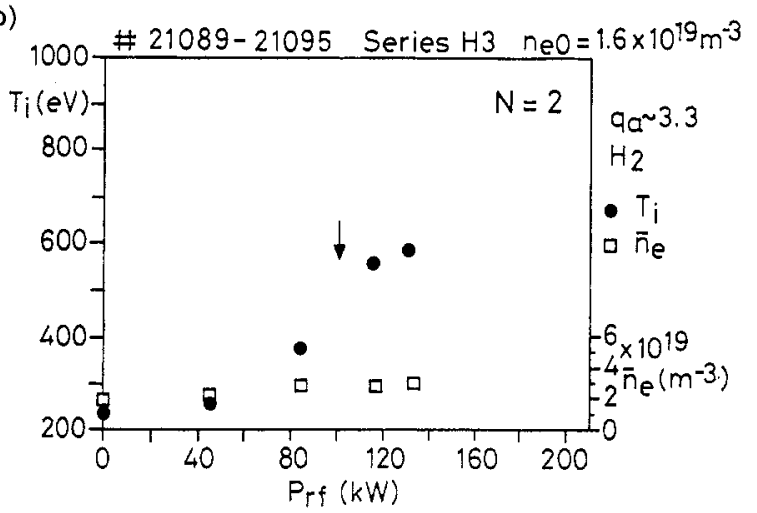

(c)

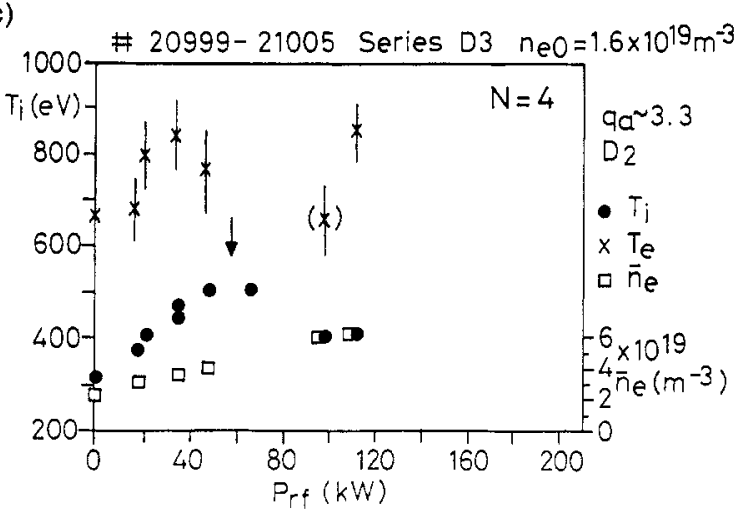

FIG. 14.-Power scan of the maximum ion temperature and density for different Alfven Wave excitation structures: (a) $N=1$, deuterium; (b) $N=2$, hydrogen; (c) $N=4$, deuterium. The electron temperatures from Thomson scattering are also shown. Vertical arrows mark the threshold of a new $r$.f. resonance at which the ion temperature does not always continue to rise with electron density. 
that a change in the spectral condition does not always have an identical effect on the ion temperature evolution, or, in other words, that the spectral effects are complicated.

This section has shown the influence of the structure of the resonance surfaces in defining the ion temperature increase. This cannot be solely ascribed to the density increase and we use this as qualitative evidence for a direct $r$.f. related power balance term. However, it cannot be used to differentiate between the plausible mechanisms listed in the last section.

\section{5. r.f. MODULATION DISCHARGES}

The possibility of a direct ion heating mechanism was further explored with a series of discharges in which the applied r.f. power was harmonically modulated by applying a sine wave perturbation to the primary pilot r.f. power stage. In the modulated perturbation technique, information is obtained from both the modulation amplitude and the phase of the plasma response to the modulation. As before, the modulated power intensity employed $(\sim 80 \mathrm{~kW})$ produced a significant plasma density change. To study the effect of a density rise, the plasma response to a harmonically modulated gas puff perturbation was also investigated.

Our analysis starts, as above, with an energy conservation equation for the ions in a central, but otherwise unspecified, region of the plasma for which we write:

$$
\mathrm{d} W_{\mathrm{i}} / \mathrm{d} t=P_{\mathrm{ei}}-W_{\mathrm{i}} / \tau_{E \mathrm{i}}+P_{A W}
$$

where $W_{\mathrm{i}}=(3 / 2) \cdot n_{\mathrm{e}} \cdot T_{\mathrm{i}}$ is the energy density of the ions, $P_{\mathrm{ei}}$ is the Artsimovitch expression for the power collisionally transferred from the electrons to the ions, $\tau_{E \mathrm{i}}$ is the ion energy confinement time, and $P_{A W}$ is a term representing a power that we have postulated to arise directly from the r.f. heating. For experiments in which the power is harmonically modulated (at an angular frequency of $\omega_{d}$ ), we assume that the central electron density and the ion temperature can be described in terms of a constant term, which does not depend on time, and a modulated time-dependent term:

$$
\begin{aligned}
& n_{\mathrm{e}}(r=0)=n_{\mathrm{c}}+n_{\mathrm{e}}^{\prime} \mathrm{e}^{-i\left(\omega_{d^{\prime}} \cdot t+\theta\right)} \\
& T_{\mathrm{i}}(\mathrm{NPA})=T_{\mathrm{i}}+T_{\mathrm{i}}^{\prime} \mathrm{e}^{-i\left(\omega_{d} \cdot t+\phi\right)}
\end{aligned}
$$

in which the primed symbols are the modulated time-dependent components and each modulated term has a separate phase. The latter are taken with respect to the applied r.f. power, which itself is taken to be described by:

$$
P_{A W}=P_{A W}+P_{A W}^{\prime} \mathrm{e}^{-i\left(\omega_{d} \cdot t+\delta\right)}
$$

with a primed term modulated component amplitude, and $\delta$, its phase relative to the perturbation.

Equations (6)-(8), together with the Artsimovitch simplification for $P_{\text {ei }}$, were substituted in equation (5) and the resulting expression was linearized with the assumption that the magnitudes of the modulated contributions are small. As above, the apparent ion energy confinement time is assumed to be constant and its value is estimated from an Ohmic discharge with similar $n_{\mathrm{e}}$ and $T_{\mathrm{i}}$. This leads to a final expression in which 
the amplitude and phase of the modulated component of the ion temperature can be obtained as a sum of three contributions arising from :

the electron-ion collisional term $(E)$

the modulation in the electron density $(N)$

the postulated direct heating term $(A)$

giving

$$
\begin{aligned}
T_{\mathrm{i}}^{\prime} \mathrm{e}^{i \phi}= & 2 \cdot P_{\mathrm{ei}} \cdot n_{\mathrm{e}}^{\prime} /\left(3 \cdot n_{\mathrm{e}}^{2} \cdot \omega_{d} \cdot s\right) \mathrm{e}^{i(\theta-\pi / 2-\psi)} \\
& -n_{\mathrm{e}}^{\prime} \cdot T_{\mathrm{i}} /\left(n_{\mathrm{e}} \cdot s\right) \mathrm{e}^{i(\theta-\psi)} \\
& +2 \cdot P_{A W /}^{\prime}\left(3 \cdot n_{\mathrm{e}} \cdot \omega_{\dot{d}} \cdot s\right) \mathrm{e}^{i(\delta-\pi / 2-\psi)}
\end{aligned}
$$

where :

$$
s^{2}=1+P_{\mathrm{ei}}^{2} /\left(n_{\mathrm{e}}^{2} \cdot \omega_{d}^{2} \cdot T_{\mathrm{i}}^{2}\right)
$$

and

$$
\tan (\psi)=1-P_{\mathrm{ei}} /\left(n_{\mathrm{e}} \cdot \omega_{d} \cdot T_{\mathrm{i}}\right)
$$

where the constants $\theta$ and $\phi$ are to be determined from the experiment.

We first use equation (9) to estimate the contribution of the additional term, using the measured $n_{\mathrm{e}}$ and ion temperature from the plasma discharge. An optimal fitting algorithm (JOYE et al., 1988), based on a statistical minimization, was used to estimate the values of the phase and amplitudes of the modulated terms, together with an estimation of the fitting error margin. For data with a good signal-to-noise, the phases were also verified graphically from a visual identification of the trace response to the applied perturbation, which was used, together with other statistical checks, as a code quality-check.

A series of modulation discharges was carried out in order to explore the plasma response in different regions of the Alfvén excited spectra. A maximum r.f. power modulation frequency of $100 \mathrm{~Hz}$ was imposed to obtain a sufficient number of N.P.A. counts within a modulation period. This frequency maximizes the number of modulation periods during a single discharge, which allows us to track the evolution of the phases and amplitudes of the modulated terms more precisely.

Most of the experiments were performed with $I_{\mathrm{p}}=120 \mathrm{kA}$ and $B_{\phi}=1.5 \mathrm{~T}$, with the programmed values of the electron density chosen in order to excite different r.f. spectral regions selectively. Although discharges were run both in hydrogen and deuterium, only deuterium discharges are reported, since no significant differences in behaviour were found. 
Figure 15 shows the temporal evolution of the ion temperature and the central electron density for three discharges. The density range during a discharge was chosen so as to cross a DAW resonance, enabling us to investigate the spectral response of the phases and amplitudes of the modulated parameters across this region.

The general characteristics are in agreement with the results of Fig. 11, in that as $n_{\mathrm{e}}$ increases, the magnitude of the modulation amplitudes decreases. The reduced modulation response at higher density, and the relatively noisy ion temperature trace, led us to concentrate on the spectral regions below and above the $(1,1)$ and $(2,0)$ DAWs, which occur at relatively low density. These were excited with $N=1$ and $N=2$ antenna structures, respectively.

A typical modulation analysis is shown in Fig. 16. The Abel inverted central electron density and the measured ion temperature are shown together with the phases of the electron modulation term and the postulated direct r.f. term required to reproduce the observed ion temperature modulation. Close to the excitation of a DAW the phases of both the ion temperature and the central density evolve smoothly between two quasi-stationary values while the relative phase of the ion temperature appears to decrease, and that of the central electron density increases.

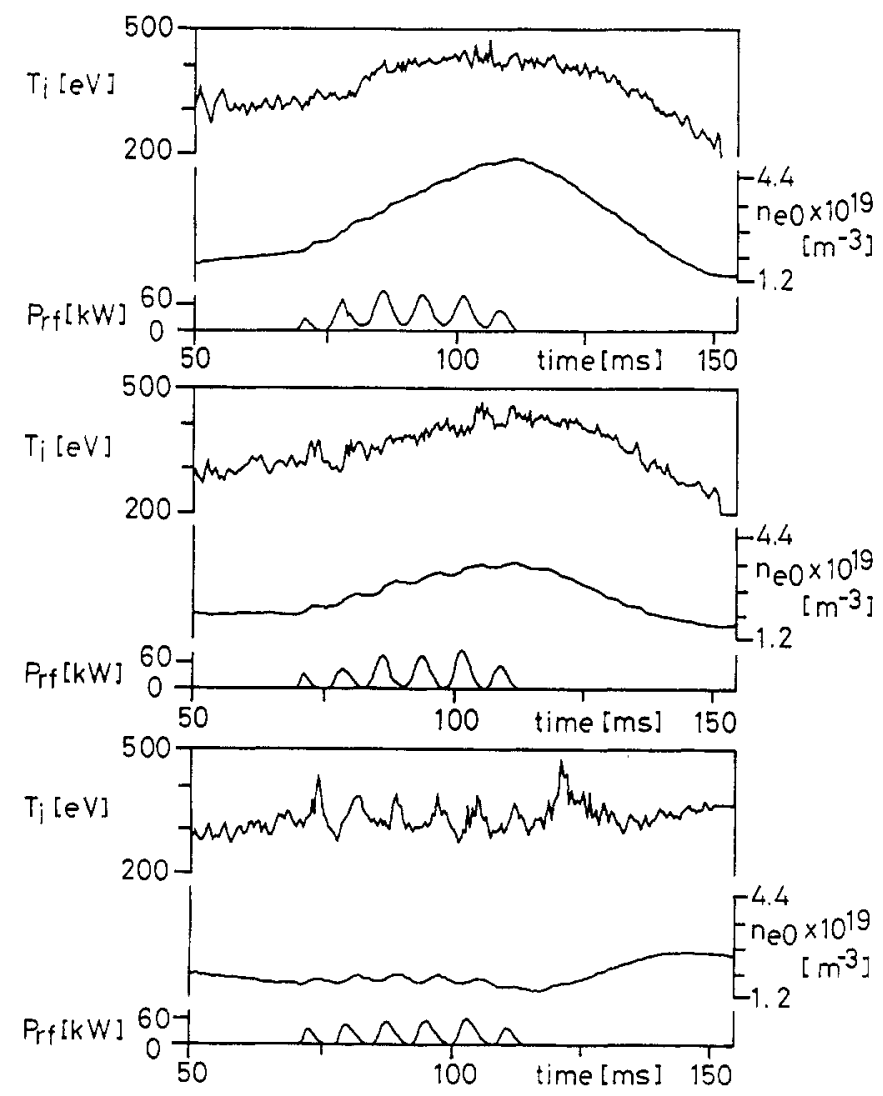

FIG. 15.-The ion temperature modulation resulting from the r.f. power modulation is shown for three plasma densities. At higher density, the modulated amplitude is clearly smaller. 


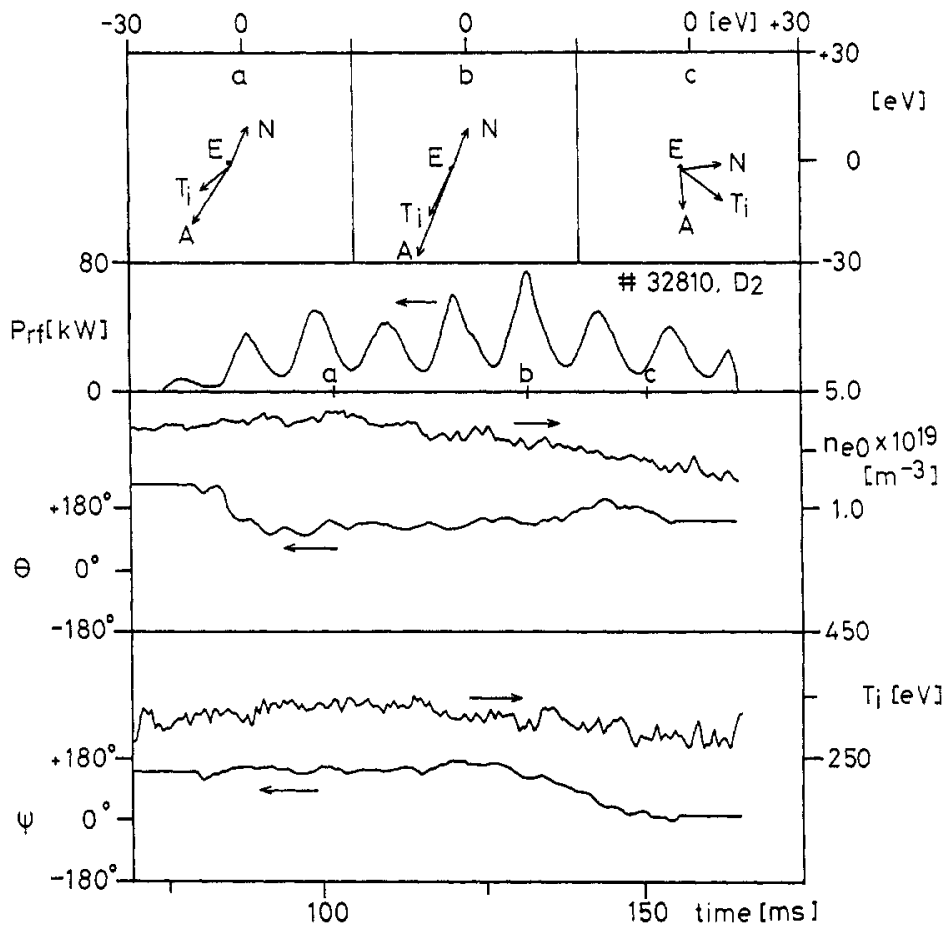

Fig. 16.-The results of the r.f. modulation experiment are shown. The evolution of the phases of the modulated components of the electron density modulation, $\theta$, and the ion temperature modulation, $\psi$, through the discharge are plotted. The components of the analysis are shown on an Argand diagram for three conditions: (a) before the DAW, (b) close to the DAW and (c) after the DAW at the times indicated on the figure (" $E$ " is the electron-ion energy transfer, " $N$ " the effect of the plasma density, " $A$ " the postulated Alfvén related term required to produce the observed ion temperature, $T_{\mathrm{i}}$ ). The phase $\psi$ is seen to slide between two quasi-stationary values across the DAW.

The results from this type of analysis may be conveniently presented in the complex plane, on an Argand diagram, where the length of the vector represents the amplitude of the modulated components, and their direction, the phases.

Figure 16 also shows the ion power balance model with Argand diagrams, before, during, and after the excitation of the $(1,1) \mathrm{DAW}$. Each known term in the power balance is plotted together with their difference, which is the additional term. It should be noted that the experimental temporal resolution does not allow us to measure whether the phase shifts occur at the DAW excitation or at the onset of a new continuum, which takes place at slightly higher values of the electron density, and thus slightly later than the DAW.

The calculated direct ion heating term shown in Fig. 16 is so large that, if it were integrated over the plasma volume, it would give a power contribution which is several times larger than the delivered r.f. power. This indicates that our power balance is either too simple or that we have neglected an important term.

In order to deduce if this is simply a density modulation effect, the ion temperature response to modulated gas puffing into Ohmic discharges was investigated. Figure 17 shows the electron density and ion temperature evolutions for a discharge with gas 


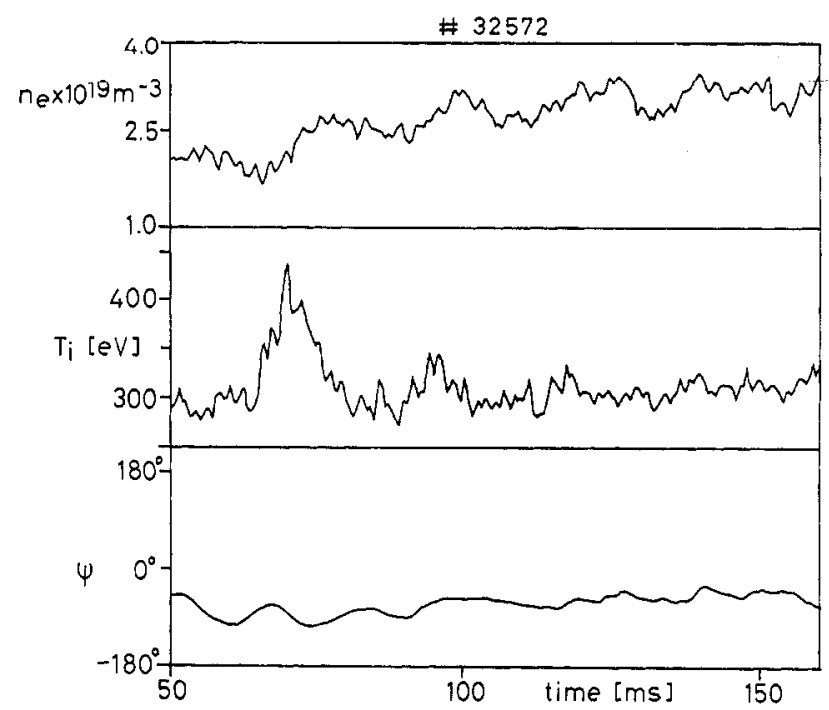

FIG. 17.-The results of the gas puff modulation in which the phase of the ion temperature response, $\psi$, does not show a slide between two values for the density range corresponding to crossing a DAW (compare with Fig. 16).

puff modulation at $100 \mathrm{~Hz}$, with the phases of the electron density and ion temperature modulated responses. When crossing the same density range as in the r.f. experiments, the phase slide observed through the DAW was not seen, but the phase of the ion temperature response relative to the central density modulation is different from the r.f. modulation case.

It is informative to display a simplified analysis which makes no assumptions about the heating mechanism. Figure 18 shows the r.f. and gas puff discharges, again in Argand diagram form, on the same scale. In order to highlight phase changes, the central electron density was always chosen as the reference, i.e. along the real axis. The electron density modulation (units of $\mathrm{m}^{-3}$ ), the ion temperature modulation (units of eV), and the deduced $P_{\mathrm{ci}}$ modulation [Equation (2), units of $\mathrm{J} \mathrm{s}^{-1} \mathrm{~m}^{-3}$ ] are shown with the modulation in the time derivative of $W_{\mathrm{i}}$ (units as for $P_{\mathrm{ei}}$ ) using equation (4) and :

$$
\mathrm{d} W_{\mathrm{i}}^{\prime} / \mathrm{d} t=j \omega W_{\mathrm{i}}^{\prime}
$$

which is achieved by multiplying the deduced magnitude of $W_{\mathrm{i}}^{\prime}$ by $\omega$ and rotating the Argand vector $+90^{\circ}$ (anti-clockwise). The axis of the modulating function, i.e. the r.f. power or the gas valve voltage, are also shown for comparison. Finally, the error is represented by a circle at the tip of the vector which includes the error in the amplitude and phase found from the code. Thus, during the experiment, the represented parameters are spinning at an angular frequency $\omega$ about the origin.

Before describing the experimental data, a simple example may help. If the modulation period was far lower than any of the relevant plasma time constants, the ion temperature modulation, and the density modulation would be expected to be in 


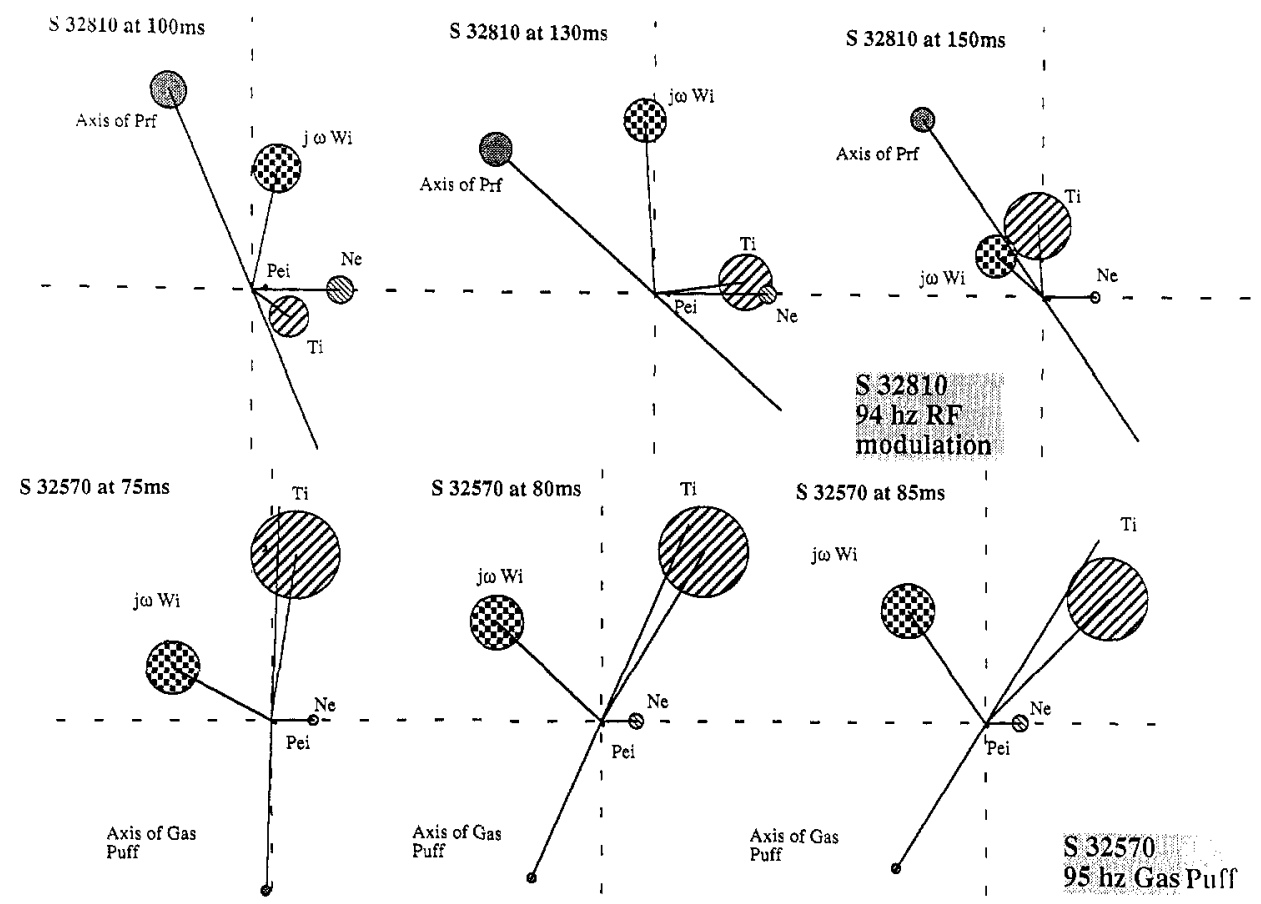

FIG. 18.-A comparison between the r.f. and Gas Puff induced density modulation discharges. The terms are represented in Argand diagram form with a circle at their tip, representing the statistical error in the measurement. The phases are all relative to the density modulation, fixed on the real axis. The units are: density modulation $\left(N_{\mathrm{e}}\right)$ in $\mathrm{m}^{-3}$; ion temperature modulation $\left(T_{\mathrm{i}}\right)$ in electronvolts. The modulated electron-ion power transfer $\left(P_{\mathrm{ei}}\right)$ and the modulated ion energy $\left(W_{\mathrm{i}}\right)$ are both in $\mathrm{J} \mathrm{s}^{-1} \mathrm{~m}^{-3}$ (same scale). The phase and relative amplitude of the modulating function is also shown.

phase, i.e. aligned, with the modulating function. Thus $P_{\mathrm{e} i}^{\prime}$ would also be aligned and $j \omega W_{i}^{\prime}$ would be at $90^{\circ}$ to the rest of the vectors. For our experimental data this is far from the case.

The most important observation from Fig. 18 is that r.f. and gas puff modulation discharges are not similar, and there would be little point in trying to use the gas puff data to account for the density modulation in the r.f. case. For the r.f. discharge, the phase slide of the ion temperature response across the DAW is very clear, and for the gas puff, the response is different, but mostly constant, through the discharge. We may conclude that with our current limitations on the pulse duration, and thus modulation frequency, we are already at a frequency where some plasma time constants play an important role in determining the ion power balance.

An interpretation of our observations as a modulation of the ion energy confinement time, $\tau_{E \mathrm{i}}$ (so far taken to be a constant), would also lack sufficient degrees of freedom to account for these differences. The simplifications made in this model are thus found to be too inexact to obtain quantitative coefficients, and more stationary state plasma conditions, with a range of modulation frequencies, are required to find the critical plasma time constants and determine if the magnitude of this enhanced ion temperature response is maintained. The frequency at which the phase of the modulated response turns also allows a determination of how many processes are involved in the 
transfer from modulated perturbation to response, i.e. in this case the modulated ion temperature.

In both experiments, with a relatively slow density modulation, there is an unexplained phase difference between the density and the ion temperature modulation. The lack of model agreement with r.f. modulation is not surprising in the light of the gas puffing result, but an empirical correction to the r.f. model, using the gas puff data, would be far more difficult to construct than that described for the unmodulated r.f. discharges.

This approach has again found a clear qualitative difference between the r.f. heating pulse and the gas puff density increase. Radio frequency spectral effects have again been observed, indicating that the r.f. wave field has a direct effect on the ion temperature.

\section{SUMMARY AND CONCLUSIONS}

This paper set out to investigate the ion temperature in the presence Alfven Wave Heating, with the same order of power as the Ohmic Heating. The launched power is taken as the difference between the power dissipated in the r.f. antenna with and without the plasma. The analysis has been made extremely difficult by the electron density rise associated with the r.f., which requires that many time-dependent terms be included in the ion power balance. The electron density rise was modelled by a time-dependent ion loss term for a non-stationary Ohmic discharge. Good agreement was found for discharges in which a rapid change in the density was produced with the gas valve.

The ion temperature evolution during the electron density rise induced by the r.f. heating did not agree with this model, which led us to consider a direct effect of the r.f. on $T_{\mathrm{i}}$, which was not theoretically expected. This was further investigated using r.f. experiments in which the r.f. power was harmonically modulated, the magnitude and phase of the ion temperature evolution produced, and the electron density, being measured relative to a $100 \mathrm{~Hz}$ r.f. modulation.

The change in the electron-ion power transfer and the associated density change were accounted for and the remainder necessary to account for the observed ion temperature was calculated. Although tending to confirm the need for a term which could be assigned directly to the r.f., the calculated size of this term was unreasonably high.

The effect of a density modulation was investigated with a $100 \mathrm{~Hz}$ modulated gas puff experiment. A significant, but different, discrepancy was found. An increase in our defined apparent ion energy confinement time could be used to explain the magnitude of the ion temperature modulation, but the effect is not sustained over the rest of the discharge and more information is required to examine the contributions to this simplified term. In any case, this analysis showed a clear difference between the Ohmic and r.f. experiments, supporting the conclusion that the r.f. has some direct influence on the ion temperature.

The effect of strong spectral features like the DAW has also been described, which again lead us to conclude that there is some direct effect on the ion temperature. It was found that the increase in the ion temperature was greatest, for a given r.f. power, just before the advent of a new continuum resonance layer. This corresponds to the present resonance layer being near the plasma edge, and a new, less efficient, con- 
tinuum resonance layer starting in the plasma centre. This implies that the heating is not associated with centrally located resonance which accounts for the majority of the r.f. power after the threshold. The intuitive idea of the ion heating associated with an r.f. resonance in the plasma center is not supported, but without a theoretical or measurable power deposition, this is difficult to pursue.

Since the electron density rise with Alfvén Heating is not yet understood, it could still be argued that the postulated direct term could be solely due to a difference in the dynamics of the r.f. and gas puff, which was for instance seen in the modulation experiments. This, however, cannot also explain the discontinuities in the $T_{\mathrm{i}}$ behaviour coincident with strong spectral r.f. features like the DAW. We thus conclude that ion temperature is in some way directly influenced by the r.f., although we cannot identify the term responsible.

Acknowledgements-We would like to thank all the members of the TCA team for the machine operation and diagnostic information. This work is partially supported by the Fonds National Suisse de la Recherche Scientifique.

\section{REFERENCES}

Afrosimov V. V. et al. (1975) Sov. Phys. Tech. Phys. 20, 1.

ApPert K. et al. (1986) Proc. IAEA, Kyoto, Invited Paper.

Chemtham A. D., Heym A., Hofmann F., Hruska K., Keller R., Lietti A., Lister J. B., Pochelon A., Ripper H., Simik A. and Tuszel A. (1981) Proc. 11 th Symp, on Fusion Technology.

Collins G. A., Hofmann F., Joye B., Keller R., Leitti A., Lister J. B. and Pochelon A. (1986) Physics Fluids 29, (7).

Behn R., Collins G. A., Lister J. B. and Weisen H. (1987) Plasma Phys. Contr. Fusion 29, 75.

Behn R., De Chambrier A., Collins G. A., Duperrex P.-A., Heym A., Moret J.-M., Nowak S., O'Rourke J., Pochelon A. and Simm W. C. (1984) Plasma Phys, 26, 173.

Besson G., De Chambrier A., Collins G. A., Joye B., Lietti A., Lister J. B., Moret J. M., Nowak S., Simm C. and Weisen H. (1986) Plasma Phys. Contr. Fusion 28, 1291.

De Chambrier A. (1987) Doctoral Thesis, EFP Lausanne, No. 712

Joye B., Lister J. B., Moret, J.-M,, Pochelon A. and Simm W. C. (1988) Plasma Phys. Contr. Fusion 30,743 .

Joye B., Marmillod PH. and Nowak S. (1986) Rev. Scient. Instrum. 57, (19). 